\title{
Innominate artery dissection during cerebral perfusion. The exception that proves the rule.
}

\author{
Antonio Piperata ${ }^{1}$, Tomaso Bottio ${ }^{2}$, Martina Avesani $^{3}$, and Gino Gerosa ${ }^{1}$ \\ ${ }^{1}$ Affiliation not available \\ ${ }^{2}$ University of Padua Medical School, \\ ${ }^{3}$ Universita degli Studi di Padova Dipartimento di Medicina
}

January 16, 2021

\begin{abstract}
Bilateral antegrade selective cerebral perfusion has the undisputed advantage of being more physiological and theoretically ensuring complete perfusion of the whole brain. However, it requires longer execution times and manipulation of the epiaortic vessels. On the other hand, unilateral selective cerebral perfusion (u-ASCP) avoids the vessels manipulation, placement of catheters into the ostia of the great vessels which clutters the operative field and incurs both atherosclerotic and air embolism risk. Neverthless, an ongoing debate about which technique yields the best clinical outcomes is still open.
\end{abstract}

\section{Innominate artery dissection during cerebral perfusion.}

The exception that proves the rule.

Antonio Piperata $^{1^{*}}, \mathrm{MD}$; Tomaso Bottio ${ }^{1^{*}}, \mathrm{MD}, \mathrm{PhD}$; Martina Avesani ${ }^{1}$, MD; Gino Gerosa ${ }^{1}$, MD.

* The first two authors equally contributed.

${ }^{1}$ Department of Cardiac, Thoracic, Vascular Sciences and Public Health, University of Padua, via N. Giustiniani, 2, 35128 Padova, Italy

Corresponding Author: Antonio Piperata,

Via Giustiniani, 2; 35128 Padova, Italy

Phone: +39 3405205163

E-mail:a.piperata88@gmail.com

Key words: Aorta and great vessels

Word count : 421

The authors do not have any conflict of interest to declare.

We carefully read the recent paper by Williams et al. (1) on the innominate artery dissection during antegrade selective cerebral perfusion (ASCP) through right axillary artery.

ASCP is considered the safest method of brain protection during aortic arch surgery (2). It can be employed to perfuse both cerebral hemispheres (bilateral cerebral perfusion) or just one (unilateral cerebral perfusion). Currently available evidence supports that both strategies are safe and guarantee satisfactory efficacy in terms of brain protection (3). Neverthless, an ongoing debate about which technique yields the best clinical outcomes is still open. 
Bilateral antegrade selective cerebral perfusion (b-ACP) has the undisputed advantage of being more physiological and theoretically ensuring complete perfusion of the whole brain. However, it requires longer execution times, manipulation of the vessels and often blind insertion of endoluminal cannulae. On the other hand, unilateral selective cerebral perfusion (u-ASCP) avoids the vessels manipulation, placement of catheters into the ostia of the great vessels which clutters the operative field and incurs both atherosclerotic and air embolism risk. Regarding u-ASCP, few data is still available in comparison to b-ACP, but the results look promising. In 2015 Angeloni et al. (4) published a systematic review and meta-analysis comparing u-ASCP and b-ASCP. Although they concluded that there is no difference between two techniques, a clear trend in favour of u-ASCP was showed, since all the absolute values were lower than b-ASCP. Indeed, a mortality rate of $9.8 \%$, a PND rate of $6.9 \%$ and a TND rate of $9.3 \%$, were reported for b-ASCP, while for u-ASCP they were $7.6 \%, 5.8 \%$, and $6.5 \%$, respectively. Following the growing experience and the good results obtained in aortic surgery, an increasing number of centres have begun to adopt u-ASCP, highlighting the suitability of this strategy as a key to reduce neurological complications, thanks to its simplicity and its lower manipulation of the arch branch vessels $(5,6)$.

The case described by Williams and colleagues (1) suggests few more considerations.

Firstly, the importance of avoiding the manipulation of epiarotic vessels during aortic surgery of both aortic aneurysm and dissections. Secondly, this case can enrich the literature on this topic, supporting the safety of u-ASCP also in complicated scenarios. Thirdly, cerebral alterations during aortic arch surgery are generally attributed to the use of cardiopulmonary bypass even if the cause of cerebral injury is probably multifactorial, including the heterogeneity of this population of patients and the surgical procedures themselves.

Lastly, as Williams et al. (1) have explained, only a full and technological monitoring can help to recognized and promptly treat any complications.

\section{REFERENCES}

1. Williams DM, Masuno K, Kanchuger MS, Hisamoto K. Innominate artery dissection due to selective cerebral antegrade perfusion. J Card Surg. 2021 Jan 5. doi: 10.1111/jocs.15290. Epub ahead of print. PMID: 33403684.

2. Di Eusanio M, Schepens MA, Morshuis WJ, Dossche KM, Di Bartolomeo R, Pacini D, Pierangeli A, Kazui T, Ohkura K, Washiyama N. Brain protection using antegrade selective cerebral perfusion: a multicenter study. Ann Thorac Surg. 2003 Oct;76(4):1181-8; discussion 1188-9. doi: 10.1016/s00034975(03)00824-5. PMID: 14530009.

3. Misfeld M, Leontyev S, Borger MA, Gindensperger O, Lehmann S, Legare JF, Mohr FW. What is the best strategy for brain protection in patients undergoing aortic arch surgery? A single center experience of 636 patients. Ann Thorac Surg. 2012 May;93(5):1502-8. doi: 10.1016/j.athoracsur.2012.01.106. Epub 2012 Apr 4. PMID: 22480393.

4. Angeloni E, Melina G, Refice SK, Roscitano A, Capuano F, Comito C, Sinatra R. Unilateral Versus Bilateral Antegrade Cerebral Protection During Aortic Surgery: An Updated Meta-Analysis. Ann Thorac Surg. 2015 Jun;99(6):2024-31. doi: 10.1016/j.athoracsur.2015.01.070. Epub 2015 Apr 16. PMID: 25890664.

5. Norton EL, Wu X, Kim KM, Patel HJ, Deeb GM, Yang B. Unilateral is comparable to bilateral antegrade cerebral perfusion in acute type A aortic dissection repair. J Thorac Cardiovasc Surg. 2020 Sep;160(3):617-625.e5. doi: 10.1016/j.jtcvs.2019.07.108. Epub 2019 Sep 5. PMID: 31587891; PMCID: PMC7061338.

6. Zierer A, Risteski P, El-Sayed Ahmad A, Moritz A, Diegeler A, Urbanski PP. The impact of unilateral versus bilateral antegrade cerebral perfusion on surgical outcomes after aortic arch replacement: a propensity-matched analysis. J Thorac Cardiovasc Surg. 2014 Apr;147(4):1212-7; discussion 1217-8. doi: 10.1016/j.jtcvs.2013.12.022. Epub 2014 Jan 2. PMID: 24507981. 